\title{
To Reviews on Embryonic Stem Cells and Germ Line
}

\author{
Norio Nakatsuji \\ Department of Development and Differentiation, Institute for Frontier Medical Sciences, Kyoto University, \\ 53 Kawaracho, Shogoin, Sakyo-ku, Kyoto 606-8507, Japan
}

Establishment of human pluripotent stem cell lines, which include embryonic stem (ES) and embryonic germ (EG) cell lines, triggered widespread attention and pointed out their unlimited potential for future regenerative medicine. To understand the unique characteristics of pluripotent cells, one should look at the cell lineages and germ line development in mammalian embryogenesis, which is the main focus of the following reviews.

For many years, mouse pluripotent stem cell lines have been important tools for basic biology to investigate cell differentiation and to carry out gene targeting for analysis of gene function in mice. ES cell lines are derived from the inner cell mass of blastocysts, while EG cell lines are derived from primordial germ cells (PGCs). PGCs originate in the epiblast, become recognizable in the posterior extraembryonic region at the primitive streak stage, then migrate to the fetal gonads and stop proliferation when taking different

\footnotetext{
Department of Development and Differentiation, Institute for Frontier Medical Sciences, Kyoto University, 53 Kawaracho, Shogoin, Sakyo-ku, Kyoto 606-8507, Japan.

Tel: +81-75-751-3808, Fax: +81-75-751-3890

E-mail: nnakatsu@frontier.kyoto-u.ac.jp
}

fates according to the sex of the fetus. Pluripotency of the ES and EG cells can be demonstrated by differentiation into various cell types in culture or teratomas, and also by the production of chimeric mice, in which ES cells contribute to all tissues including the germ line. Therefore, germ cells and pluripotent cells have unique relationships to be investigated.

An exciting new aspect is the reprogramming capacity of the cell and nucleus. Recent reports have indicated that various types of somatic stem cells can change their differentiation repertoire in still unclear circumstances. On the other hand, animal cloning indicated that nuclei of differentiated somatic cells could be reprogrammed in unfertilized eggs to restart embryogenesis. In addition, cell fusion experiments between ES or EG cells with differentiated somatic cells resulted in reprogramming of the somatic nucleus. Such various types of reprogramming may be related to each other and involve common factors and regulative mechanisms, which should be very important in both basic and applied sciences. 